\title{
Research Paper \\ Comparing the Effect of Cold and Warm Stimulation of Hoku Point on Pain Caused by Removal of External Fixators Pin in Patients with Hand Fractures
}

\author{
Nasrin Rajabi ${ }^{1}$, Maasoumeh Barkhordari-Sharifabad ${ }^{2, *}$, Alireza Abedi ${ }^{3}$, Hossein Fallahzadeh \\ ${ }^{1}$ M.Sc. Student of Critical Care Nursing, Department of Nursing, School of Medical Sciences, Yazd Branch, Islamic Azad \\ University, Yazd, Iran \\ 2 Ph.D. in Nursing, Assistant Professor, Department of Nursing, School of Medical Sciences, Yazd Branch, Islamic Azad \\ University, Yazd, Iran \\ ${ }^{3}$ Ph.D. in Orthopedic, Kashan University of Medical Sciences, Kashan, Iran \\ ${ }^{4}$ Ph.D. in Biostatistics, Department of Biostatistics and Epidemiology, School of Health, Shahid Sadoughi University of Medical \\ Sciences and Health Services, Yazd, Iran \\ * Corresponding author: Maasoumeh Barkhordari-Sharifabad, Ph.D. in Nursing, Assistant Professor, Department of Nursing, \\ School of Medical Sciences, Yazd Branch, Islamic Azad University, Yazd, Iran. E-mail: barkhordary.m@gmail.com \\ How to Cite this Article: \\ Rajabi N, Barkhordari-Sharifabad M, Abedi A, Fallahzadeh H. Comparing the Effect of Cold and Warm Stimulation of Hoku Point on Pain Caused \\ by Removal of External Fixators Pin in Patients with Hand Fractures. J North Khorasan Univ Med Sci. 2020;6(4):39-48. \\ DOI: 10.29252/ijrn-06405
}

\begin{tabular}{l} 
Received: 24 Sep 2019 \\
Accepted: 09 Mar 2020 \\
\hline Keywords: \\
Hoku Point Stimulation \\
Cold \\
Warm \\
Pain \\
External Fixator \\
\hline C 2020 Iranian Journal of \\
Rehabilitation Research in Nursing
\end{tabular}

Rehabilitation Research in Nursing

\begin{abstract}
Introduction: One of the most common complaints in patients during the pin is removed from the metacarpal bone fracture is pain. Topical warm and cold therapy have effects on the skin so that it can relieve pain. On the other hand, according to traditional Chinese medicine, the Hoku point is the most crucial pain point in the body. This study aimed to compare the effect of cold and warm stimulation of Hoku point on pain Caused by Removal of External Fixators pin in patients with hand fractures.

Methods: This randomized clinical trial study was performed on 109 patients with metacarpal finger fracture with an external pin referring to the emergency department of Shahid Beheshti Hospital in Kashan in 2019. Patients were divided into three groups through block randomization: Huko point massage with ice, Huko lead massage with a warm compress and routine care. The Hoku point massage was done for 10 minutes before the pin was removed. Patients' pain severity was measured by the McGill pain questionnaire immediately after removing the pin in all three groups. Data were analyzed using the Chi-square test and one-way ANOVA. Results: The mean total pain score in the Huko point stimulation group with a warm compress, ice, and routine care was 65/33 $\pm 1 / 50,31 / 21 \pm 5 / 21$, and 42/30 $\pm 4 / 93$, respectively. The results of one-way ANOVA showed a significant difference in terms of overall pain between the three groups $(\mathrm{P}<0.001)$. Post hoc test showed that the mean score of all pain and its dimensions were significantly lower in the coldstimulated Hoku point group than in the other two groups and in the Hokustimulated point-warm group considerably lower than the control group $(\mathrm{P}<0.001)$. Conclusions: Cold-stimulated Hoku point stimulation significantly attenuated pain due to external pin removal in patients with fractures compared to Hoku-stimulated point-warm or routine care. Therefore, considering the cheapness, applicability, and safety of this method, it is recommended to plan nurses' training in applying alternative medicine approaches, including stimulation of Hoku point.
\end{abstract}




\section{Extended Abstract}

\section{OBJECTIVE}

$\mathrm{O}$ ne of the most common complaints in patients during the pin is removed from the metacarpal bone fracture is pain (5). Topical warm and cold therapy has effects on the skin so that it can relieve pain $(9,10)$. On the other hand, according to traditional Chinese medicine, the Hoku point is the most critical pain point in the body (11). This study aimed to compare the effect of cold and warm stimulation of Hoku point on pain Caused by Removal of External Fixators pin in patients with hand fractures.

\section{METHODS}

This randomized clinical trial study was done in the emergency department of Shahid Beheshti Hospital, Kashan, Iran, from November 2018 to May 2019. The study population consisted of all patients with a metacarpal fracture with an external pin referred to the emergency department of Kashan Shahid Beheshti Hospital for Removal of External Fixators pin.

The sample size in each group was 36 patients. Inclusion criteria consist of Willingness to participate in the study, Metacarpal fracture of the finger and presence of external fixator, lack of consciousness disorder, Verbal communication ability, lack of Inflammation, and biting at the point of massage. Exclusion criteria included withdrawal during the study. Sampling was done through a convenience method and then blocked randomization. The ethics committee of Yazd Islamic Azad University was allowed to do this study (IR.IAU.KHUISF.REC.397.220), and the design of this study was recorded on the clinical trial website (NO.IRCT20181119041699N2). Before sampling, study objectives were explained to all participants, and informed consent was obtained from all participants in the study. Patients participated in the study voluntarily and freely and could be excluded at any stage of the research without any restrictions. Data collection instrument included demographic questionnaire (age, sex, marital status, education level) and 20-item McGill Pain Questionnaire on a Likert scale (each question ranged from 1 to 6); higher scores indicated a higher perception of pain .The minimum score was 20 , and the maximum was 120. The McGill Pain Questionnaire was designed by Melzac and was used on 297 patients suffering from various types of paiN (16). In Iran, Khosravi et al. (2012) reported the internal consistency coefficient of this questionnaire as 0,85 (Cronbach's alpha), and the internal consistency coefficient of all four components were above 0, 80 (17). After introducing themselves, the purpose of the study, and explaining how to complete the questionnaires and assure the study patients of the confidentiality of the answers, Patients were assessed for inclusion and exclusion criteria. After obtaining written consent from them, they entered the study.

At the beginning of the study, a demographic questionnaire for patients was filled through a selfreport method. Subsequently, patients who were continuously enrolled in the study were divided into three groups (Huko point massage with ice, Huko point massage with a warm compress, and routine care). Hoku point massage for both groups was performed by a researcher who had previously received the necessary training to perform the massage.

In this study, to massage the Huko point with ice, the researcher placed the bottom of a plastic bottle of polyethylene on the Huko point of the hand on the opposite side of the fracture (the hand that had no fracture) for 10 minutes before removing the pin .To massage the Huko point with a warm water compress, the researcher applied the bottom of the warm water bottle of polyethylene containing water at $45^{\circ} \mathrm{C}$ to the Huko end of the opposite side of the fracture (the nonfractured hand) for 10 minutes before removing the pin. Immediately after pin withdrawal and patients' condition stabilized in all three groups, the pain questionnaire was completed by interviewing patients in the room set apart to remove the external pin. Patients were followed up in a single-blind study in which Statistician has not known the type of intervention until the end of data analysis. Data were analyzed using the Chi-square test and one-way ANOVA.

\section{RESULTS}

The mean total pain score in the Huko point stimulation group with a warm compress, ice, and routine care was $65.33 \pm 1.50,31.21 \pm 5.21$, and $42.30 \pm 4.93$, respectively. The results of one-way ANOVA showed a significant difference in terms of overall pain between the three groups $(\mathrm{P}<0.001)$. Post hoc test showed that the mean score of all pain and its dimensions were significantly lower in the cold-stimulated Hoku point group than in the other two groups and in the Hokustimulated point-warm group significantly lower than the control group $(\mathrm{P}<0.001)$.

\section{CONCLUSION}

Cold-stimulated Hoku point stimulation significantly attenuated pain due to external pin removal in patients with fractures compared to Hoku-stimulated pointwarm or routine care. Therefore, considering the cheapness, applicability, and safety of this method, it is recommended to plan nurses' training in applying alternative medicine approaches, including stimulation of Hoku point. 


\section{Ethical Considerations}

The ethics committee of Yazd Islamic Azad University was allowed to do this study (IR.IAU.KHUISF.REC.397.220), and the design of this study was recorded on the clinical trial website (NO.IRCT20181119041699N2). Before sampling, study objectives were explained to all participants, and informed consent was obtained from all participants in the study. Patients participated in the study voluntarily and freely and could be excluded at any stage of the research without any restrictions.

\section{Funding}

This study had no sponsors and was conducted with a personal cost.

\section{Authors' Contributions}

Ms. Nasrin Rajabi Conducting the Intervention Program, Entering Study Data into Statistical Program and primary drafting Paper, Maasoumeh Barkhordari-
Sharifabad Submit, Follow up and revise the paper, Alireza Abedi was supervising Intervention Implementation, Hossein Fallahzadeh Statistical Analysis of Study Data.

\section{Conflicts of Interest}

The authors acknowledge that there is no conflict of interest in this study.

\section{Applicable Remarks}

This study will be useful to pain relief due to the Removal of External Fixators pin in patients with hand fractures as a cheap, low-risk, and secure method.

\section{Acknowledgments}

The researchers would like to thank the patients who collaborated with us in conducting this study, the Vice Chancellor for Research and Technology of the Islamic Azad University of Yazd, and the emergency department personnel of Shahid Beheshti Hospital in Kashan. 


\section{مقايسه تأثير تحريك نقطه هوكو با سرما و كر ما بر درد ناشى از خروج يين اكسترنال در بيمار ان با شكستگى دست}

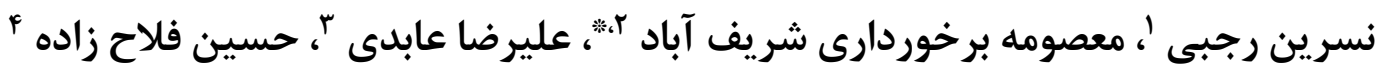

' دانشجوى كارشناسى ارشد يرستارى مراقبت ويرّ، دانشكده علوم يزشكى، واحد يزد، دانشكاه آزاد اسلامى، يزد، ايران

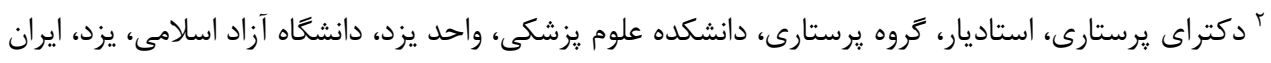

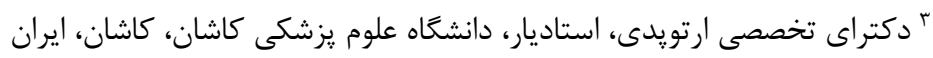

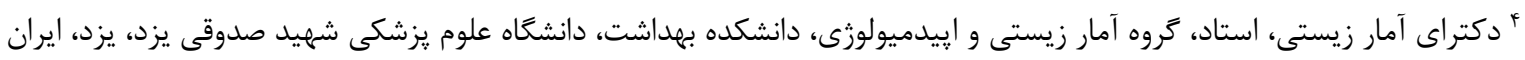
* نويسنده مسئول: معصومه برخوردارى شريف آباد، دكتراى يرستارى، استاديار، گروه يرستارى، دانشكده علوم يزشكى، واحد يزد، دانشخاه آزاد اسلامى، يزد، ايران. ايميل: barkhordary.m@gmail.com

مقدمه: يكى از شكايتهاى شايع در بيماران هنگام خروج بين از محل شكستخى استخوان متاكارٍ، درد است. استفاده از گرما و سرما درمانى موضعى

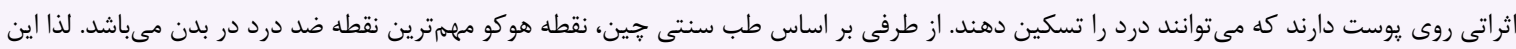

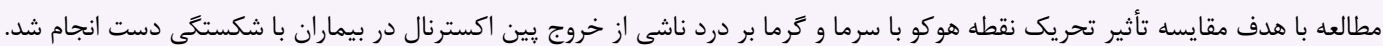

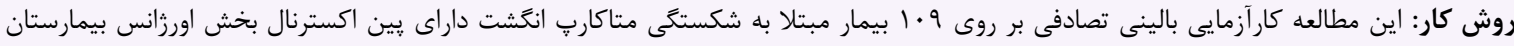

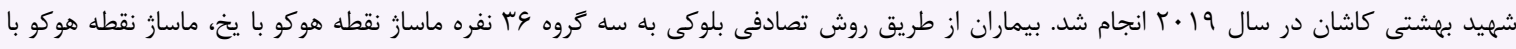

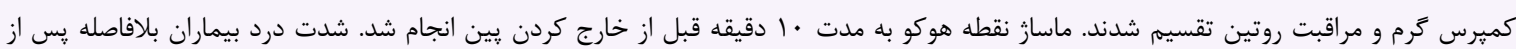

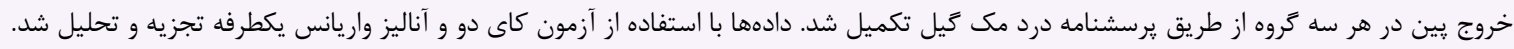

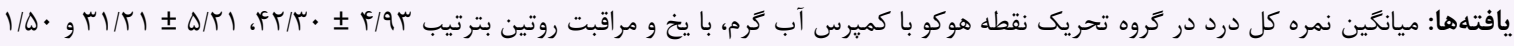

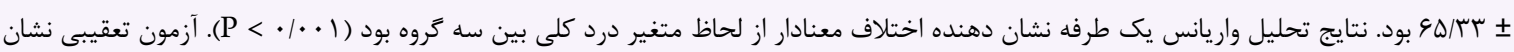

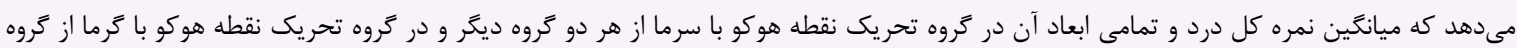

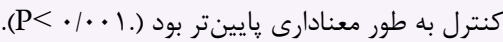

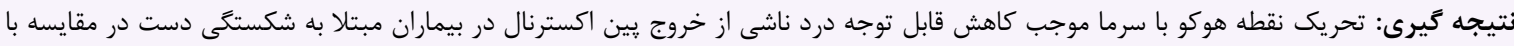

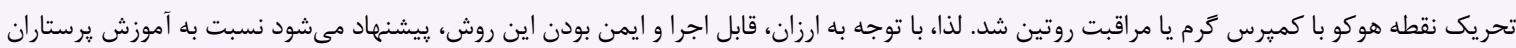

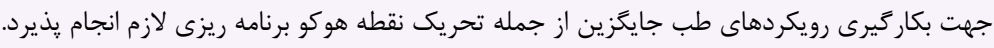

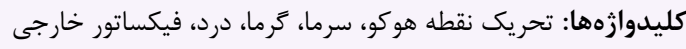

تمامى حقوق نشر براى انجمن علمى يرستارى ايران محفوظ است.

مقدمه

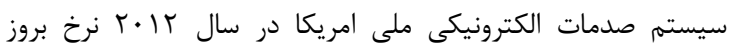

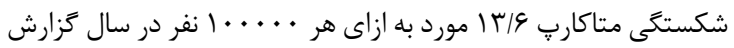

شده است [ب].

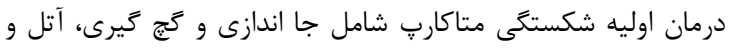
فيكس كردن مىباشد [ [ ]. يكى از روش هارى فيكس كردن استفاده از
شكستكى دست، يكى از شايعترين صدمات ارتويدى در واحدهاى

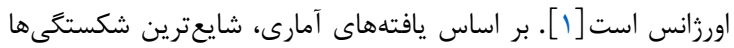

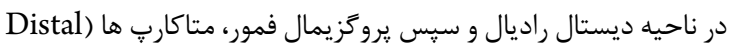

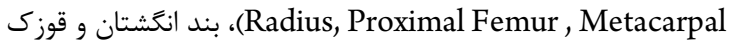

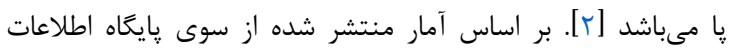


تاكنون هيج مطالعهاى در زمينه مقايسه تاثيرماساز يخ با كميرس گرم

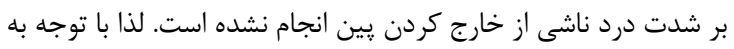
آمار بالاى بيمارانى كه دجار شكستكى متار كارِ

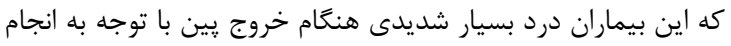

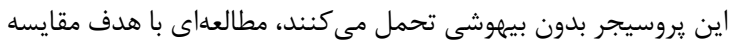

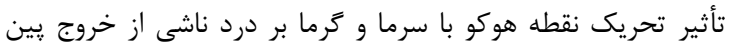

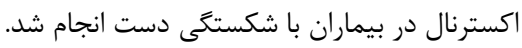

\section{روش كار}

اين مطالعه كارآزمايى بالينى تصادفى در بخش اورزانس بيمارستان

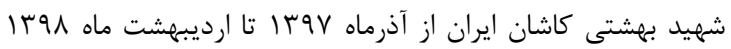

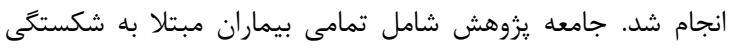

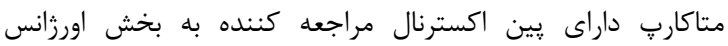

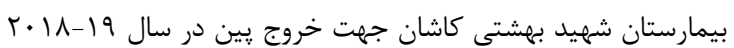
مىباشد. جهت برآورد حجم نمونه، با در نظر كرفتن خطاى نوع اول هـاء و توان

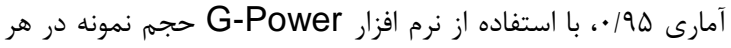

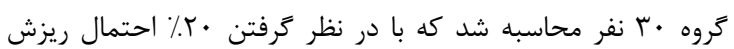

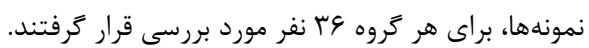

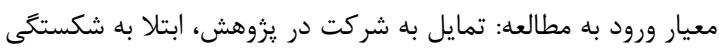

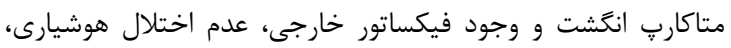

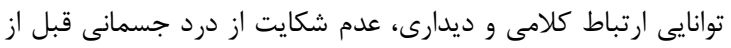

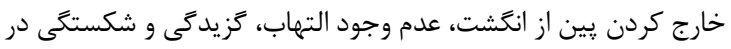

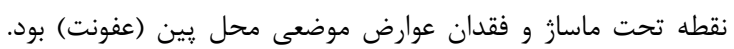

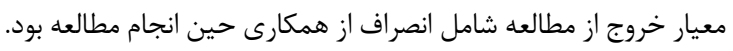

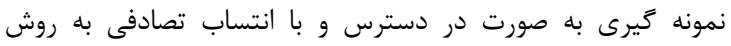

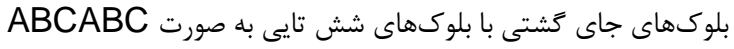

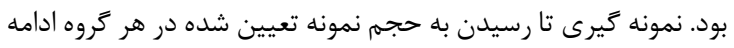

$$
\text { يافت (تصوير ()). }
$$

جهت انجام اين مطالعه از كميته اخلاق دانشكاه آزاد اسلامى برستارى (برى

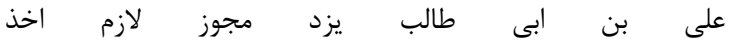

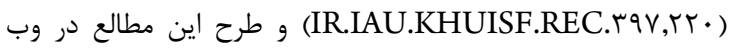

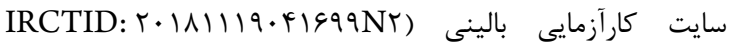
ثبت شد. قبل از نمونه كيرى اهداف مطالعه براى كليه

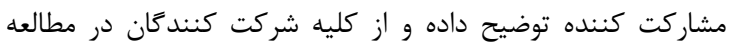

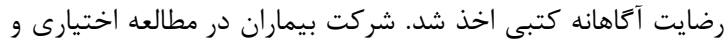

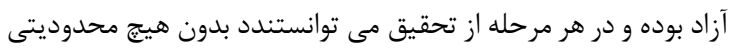

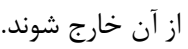
ابزار جمع آورى اطلاعات مطالعه از دو بخش تشكل شده است: بخش

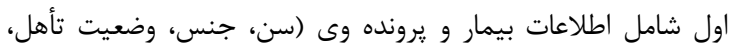

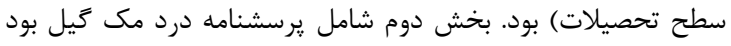

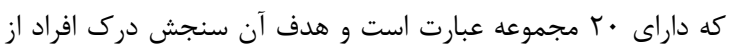

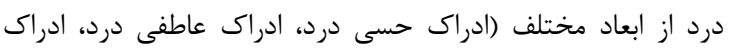

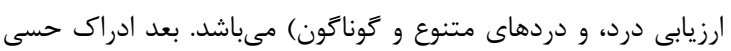

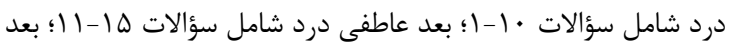

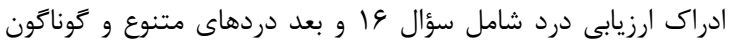

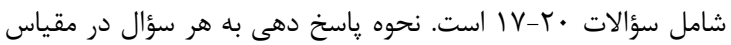

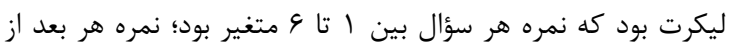

فيكساتور خارجى (external fixator) است [ع]. فيكساتور خارجى

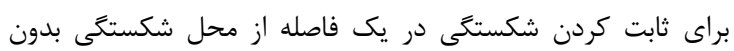

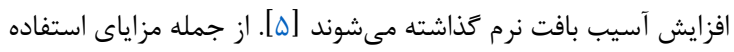
از فيكساتورهاى خارجى مى توان به حفظ طول و محور اندام شكسته ندائه

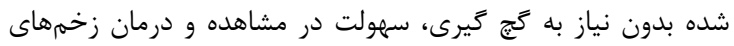

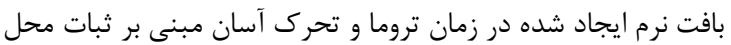
شكستخى اشاره داشت [ع].

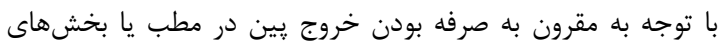

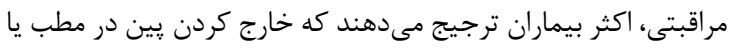

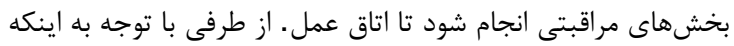

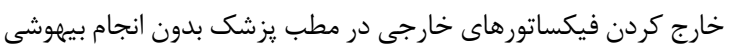

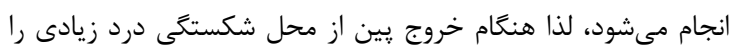

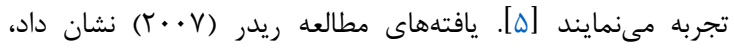

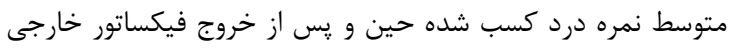

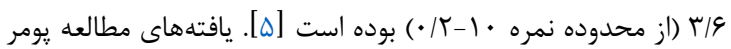

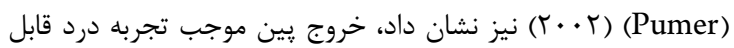

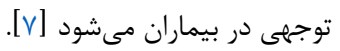

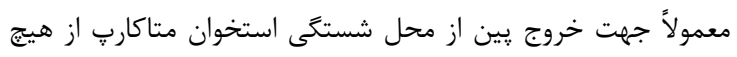

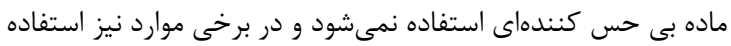

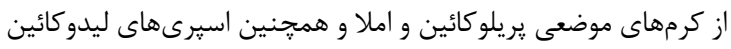

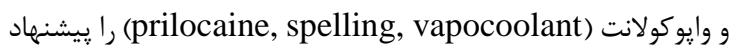
كردهاند [ه]. با توجه به عوارضى همجون انقباضات عروقى، خارش،

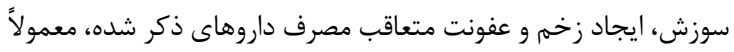

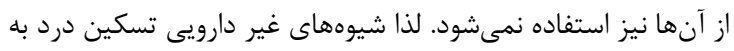

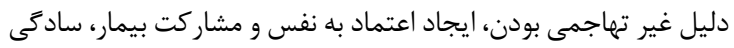

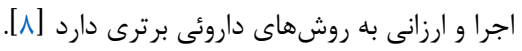

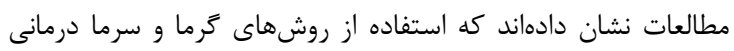

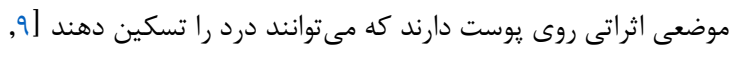

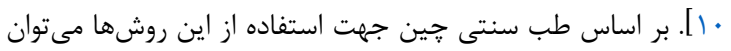

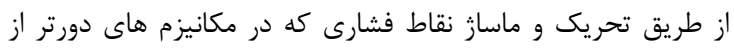

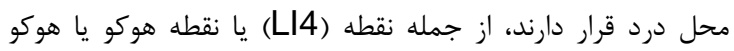

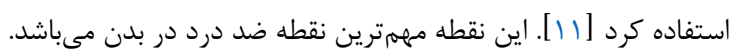

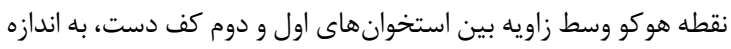

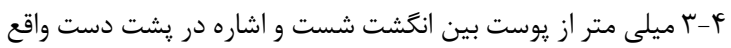

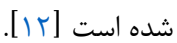
نتايج مطالعات مختلف حاكى از تأثير ماساز نقطه هو كو با گَرما و سرما

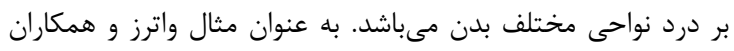

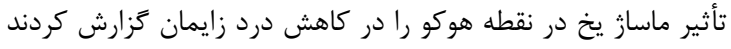

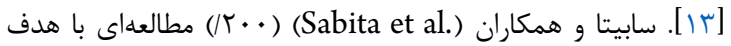

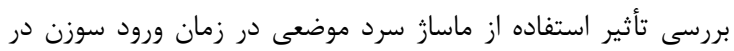

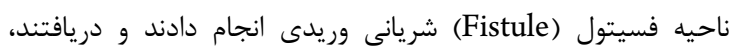

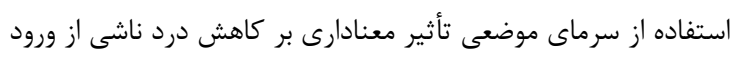

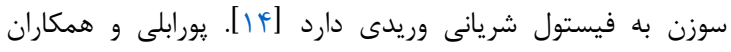

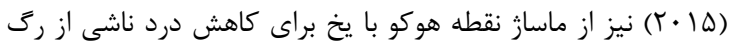

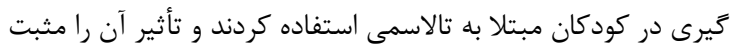

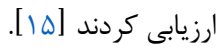
در مطالعات فوق الذكر، تأثير سرما يا كرما اغلب به صورت جداء آنانه در

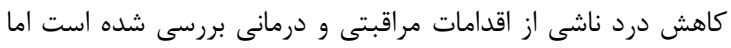


متر و ضخامت ا ميلى متر و حجم •ه ميلى متر حاوى آب با دماى

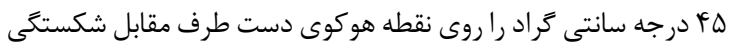

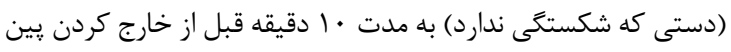

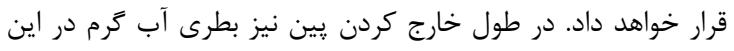

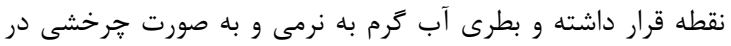

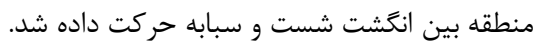

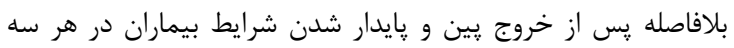

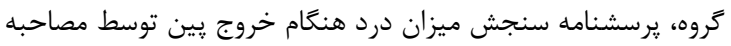

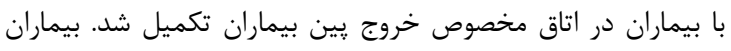

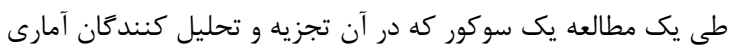

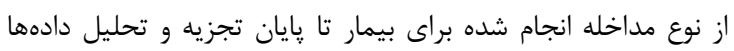
اطلاعى نداشتند، يِيخيرى شد.

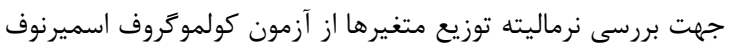

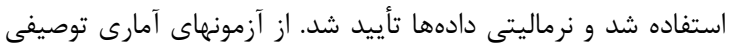

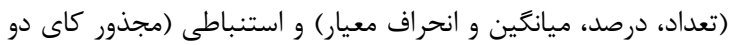
و آناليز واريانس يك طرفه) با استفاده از نرم افزار

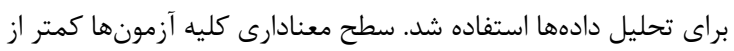

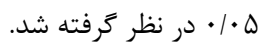

\section{يافتهها}

11 ا نفر به دليل عدم تمايل به تكميل ترسشنامه و عدم تمايل به دريافت

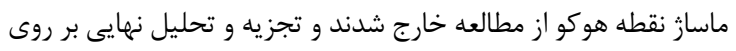
Vو نفر انجام شد (شكل ()). ميانكين سن شركت كنند

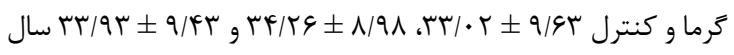

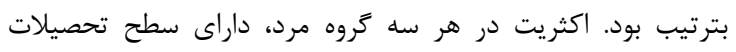

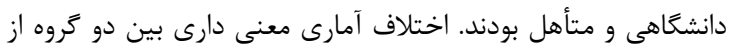

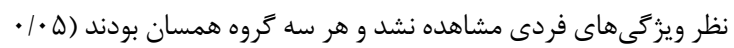

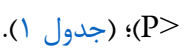
نتايج تحليل واريانس يك طرفه نشان دهنده اختلاف معنادار بين سه (جن

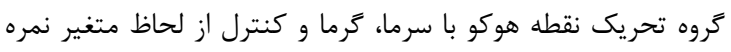

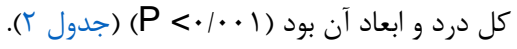

مجموع جمع نمرات سؤالات آن بعد محاسبه شد. امتياز بالاتر نشان

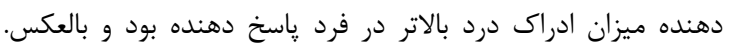

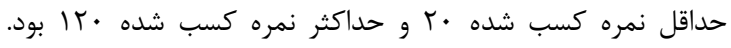

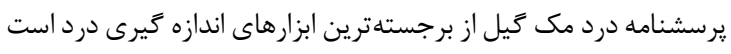

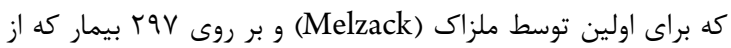

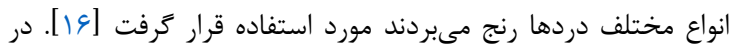

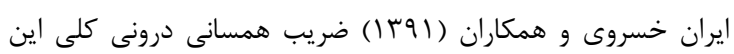

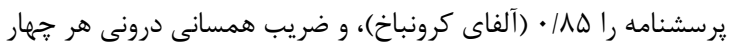

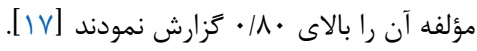

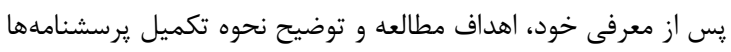

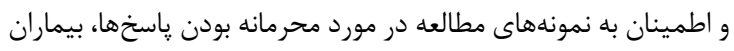

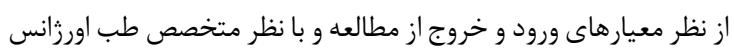

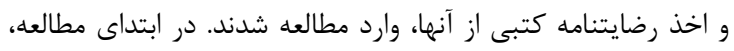

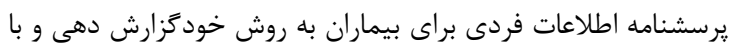

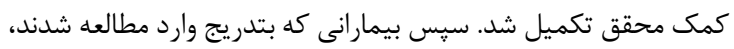

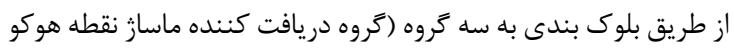

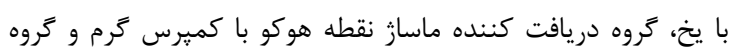

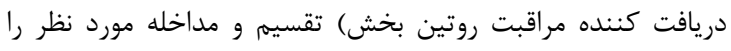

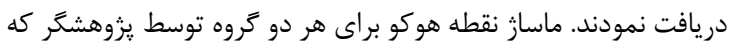

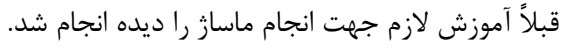

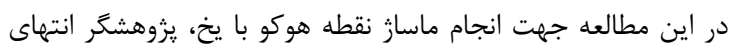

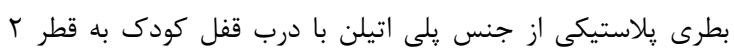

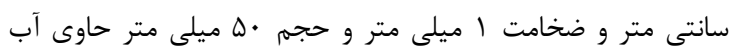

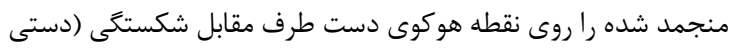

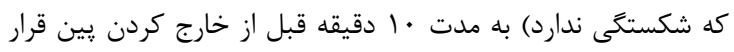

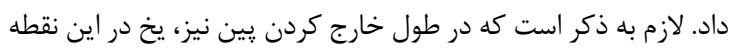

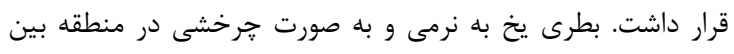

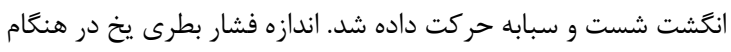

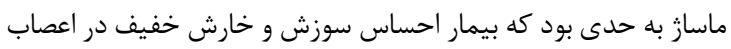

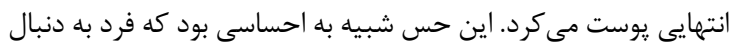
خاراندن يك ناحيه داشت.

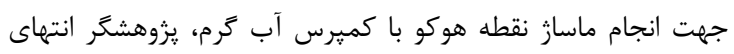

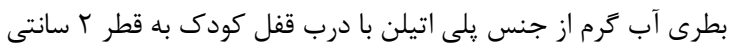

جدول ا. مشخصات دموكرافيك بيماران مبتلا به شكستىى دست در سه كروه تحريك نقطه هوكو با سرما، كَما و كتترل

\begin{tabular}{|c|c|c|c|c|}
\hline P Value & تحريك نقطه هوكو با كرما & تحريك نقطه هوكو با سرما & 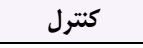 & متغيرهاى زمينهاى كروه درمانى \\
\hline \multirow[t]{3}{*}{ "*ATr } & & & & 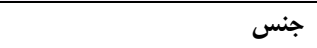 \\
\hline & $\mathbb{I r}(\kappa+\Gamma)$ & $11(r 9 / v)$ & $q(r \cdot)$ & 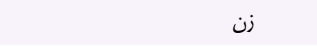 \\
\hline & $\operatorname{IV}(\Delta S / V)$ & $r(V \cdot / r)$ & $r)(v \cdot)$ & مرد \\
\hline \multirow[t]{4}{*}{ "./979 } & & & & تحصيلات \\
\hline & $r(\xi / V)$ & $1(T / V)$ & $r(\xi / V)$ & ابتدايى \\
\hline & $g(r \cdot)$ & $\wedge(r \backslash / 9)$ & $\wedge(r \& / 9)$ & دييلم \\
\hline & $r r(N / T)$ & $r \Lambda(Y \Delta / V)$ & $r \cdot(\varepsilon q / V)$ & دانشخاهى \\
\hline \multirow[t]{3}{*}{$\cdot / \Delta \mathrm{V} \Lambda$} & & & & 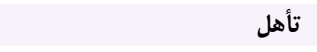 \\
\hline & $1 \cdot(r r / \Gamma)$ & $\operatorname{IV}\left(F^{\circ} \Delta / Q\right)$ & $\mathbb{I r}(\boldsymbol{x} \cdot)$ & 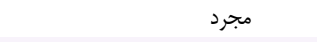 \\
\hline & $r \cdot(\Phi \& / V)$ & $r \cdot(\Delta r / l)$ & $M(\varepsilon \cdot)$ & متأهل \\
\hline "*AALT & TE/KE $\pm N / Q \Lambda$ & $r \Psi / . r \pm q / q \mu$ & $r \Psi / q \Psi \pm q / \notin \Psi$ & سن \\
\hline
\end{tabular}




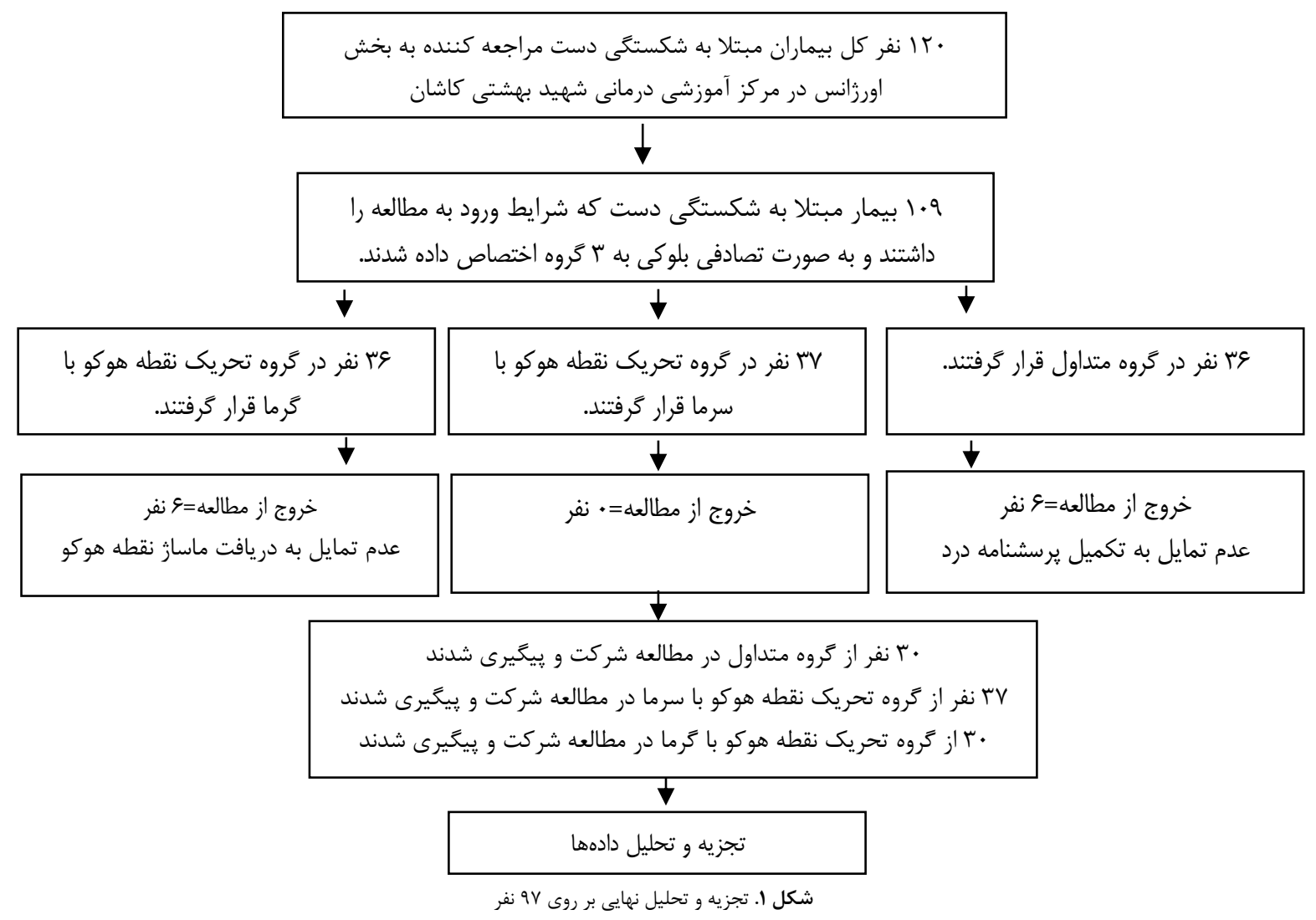

\begin{tabular}{|c|c|c|c|c|}
\hline $\mathbf{P}^{*}$ & $F$ & انحراف استاندارد & ميانكَين & تروه \\
\hline \multirow[t]{4}{*}{$<\cdot 1 \cdot \cdot 1$} & FAY/FIF & & & ادراك حسى درد \\
\hline & & $\Gamma / 1 Q$ & $r r / q$. & تحريك نقطه هوكو با گرما \\
\hline & & $r / \Delta$. & $10 / r q$ & تحريك نقطه هوكو با سرما \\
\hline & & $r / \cdot 9$ & ro/ & كنترل \\
\hline \multirow[t]{4}{*}{$<\cdot / \cdot \bullet$} & $1.9 / 948$ & & & ادراك عاطفى درد \\
\hline & & $1 / r$ & V/R & تحريك نقطه هوكو با گرما \\
\hline & & $1 / 09$ & s/19 & تحريك نقطه هوكو با سرما \\
\hline & & $t / I F$ & שT/TI & 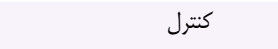 \\
\hline \multirow[t]{4}{*}{$<\cdot / \bullet 1$} & $111 \% / 99 \Delta$ & & & ادراك ارزيابى درد \\
\hline & & $1 / 1$ & $r / \varphi$. & تحريك نقطه هوكو با كرما \\
\hline & & ./VTF & $1 / 4$ & تحريك نقطه هوكو با سرما \\
\hline & & ./VGF & c/яT & 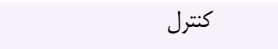 \\
\hline \multirow[t]{4}{*}{$<\cdot 1 \cdot \cdot 1$} & $98 / K \cdot 1$ & & & دردهاى متنوع و تَوناتَون \\
\hline & & $t / V T$ & १/१५ & تحريك نقطه هوكو با گرما \\
\hline & & $r / \cdot T^{c}$ & $V / \cdot \Lambda$ & تحريك نقطه هوكو با سرما \\
\hline & & $1 / 94$ & س & 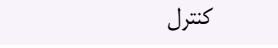 \\
\hline \multirow[t]{4}{*}{$<\cdot / \cdot \cdot 1$} & FYV $/ \Delta \mathrm{V}$ & & & ادراى كلى درد \\
\hline & & r/ar & er/T. & تحريك نقطه هوكو با گرما \\
\hline & & $\Delta / / 1$ & TI/TI & تحريك نقطه هوكو با سرما \\
\hline & & $1 / \Delta$. & SD/rT & كتترل \\
\hline
\end{tabular}


هنكام استفاده از كميرس آب كرم مىباشد [1/] كه همسو با نتايج

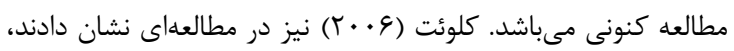

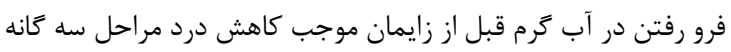

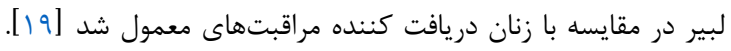

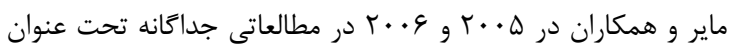

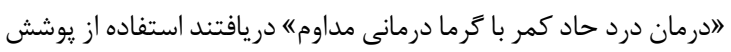

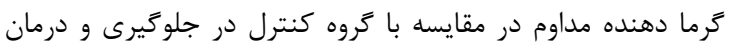

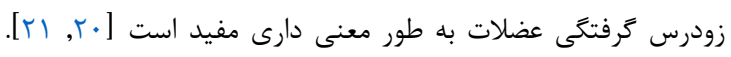

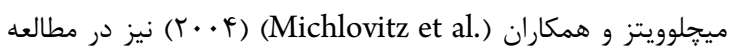

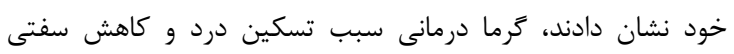

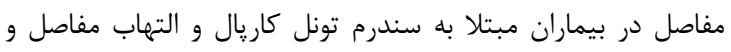

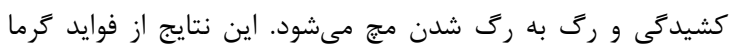

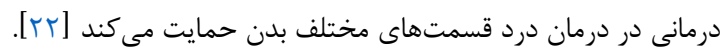
كرما موجب كاهش فعاليت فيبر كاما در عضله و و كاهش حسان حساسيت

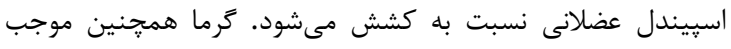

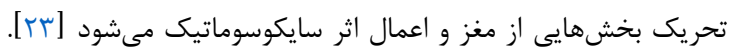

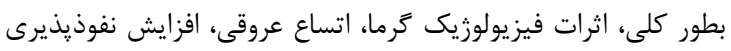

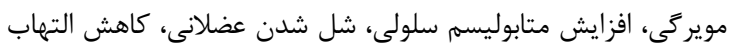

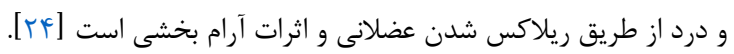

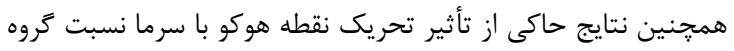

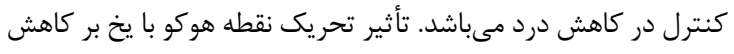

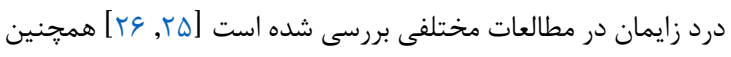

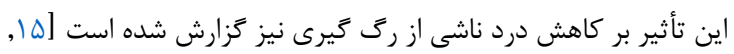

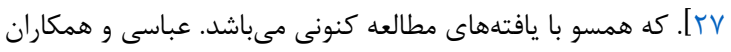

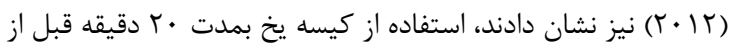

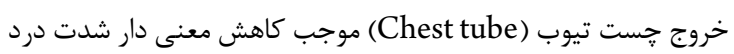

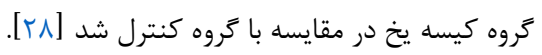

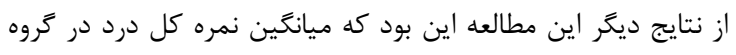

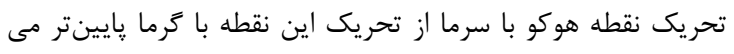

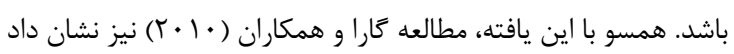

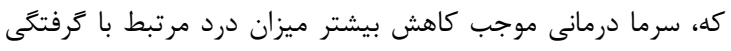

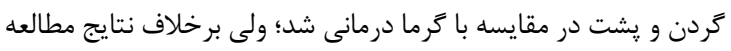

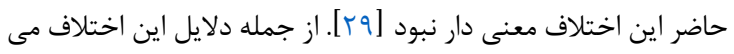

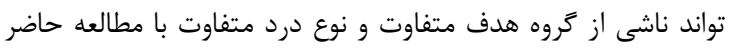

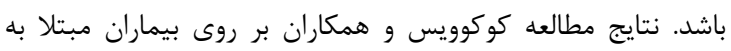

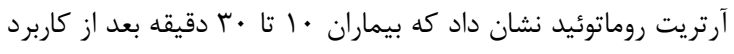

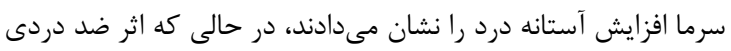

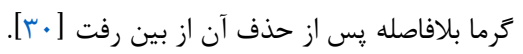

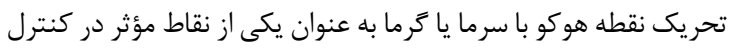

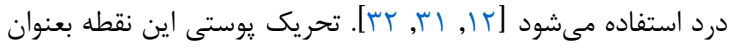

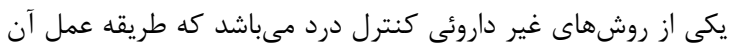

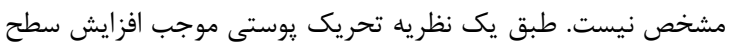

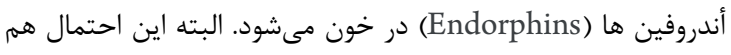

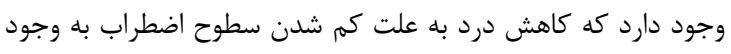

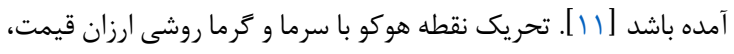

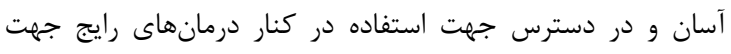

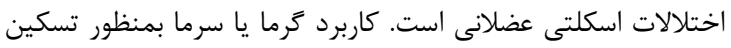

\begin{tabular}{|c|c|c|}
\hline & & هوكو با سرما، كرما و كنترل \\
\hline $\mathbf{P}$ & تفاوت ميانكَين & 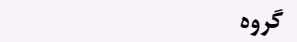 \\
\hline \multicolumn{3}{|c|}{ ادراك حسى درد } \\
\hline \multicolumn{3}{|r|}{ تحريك نقطه هوكو با سرما } \\
\hline$<\cdot / \cdot .1$ & $-N / \mu$. & تحريك نقطه هوكو با كرما \\
\hline \multirow[t]{2}{*}{$<\cdot / . .1$} & $-19 / 9$ & 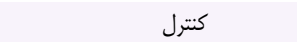 \\
\hline & & تحريك نقطه هوكو با ترما \\
\hline$<. / . .1$ & $-11 / \xi^{\mu}$ & 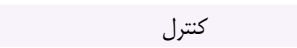 \\
\hline \multicolumn{3}{|c|}{ ادراك عاطفى درد } \\
\hline \multicolumn{3}{|r|}{ تحريك نقطه هوكو با سرما } \\
\hline$<. / . .1$ & $-1 / T V$ & تحريك نقطه هوكو با كرما \\
\hline \multirow[t]{2}{*}{$<\cdot / \cdot .1$} & $-81 \cdot V$ & 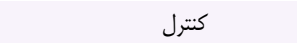 \\
\hline & & تحريك نقطه هوكو با ترما \\
\hline$<\cdot 1 \cdot .1$ & $-r / \Lambda$ & 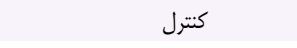 \\
\hline \multicolumn{3}{|c|}{ ادراك ارزيابى درد } \\
\hline \multicolumn{3}{|c|}{ تحريك نقطه هوكو با سرما } \\
\hline$<\cdot / . .1$ & $-1 / 19$ & تحريك نقطه هوكو با كرما \\
\hline \multirow[t]{2}{*}{$<\cdot / \cdot .1$} & $-r / T r$ & 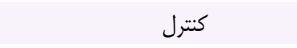 \\
\hline \multirow{2}{*}{\multicolumn{3}{|c|}{ تحريك نقطه هوكو با ترما }} \\
\hline & & \\
\hline \multicolumn{3}{|c|}{ دردهاى متنوع و توناتون } \\
\hline \multicolumn{3}{|r|}{ تحريك نقطه هوكو با سرما } \\
\hline$<\cdot / \cdot .1$ & $-r / \wedge \Delta$ & تحريك نقطه هوكو با كرما \\
\hline$<\cdot / \cdot .1$ & $-9 / 10$ & 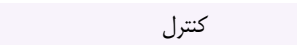 \\
\hline \multicolumn{3}{|r|}{ تحريك نقطه هوكو با ترما } \\
\hline$<\cdot / . .1$ & $-r / r \cdot$ & 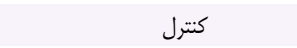 \\
\hline \multicolumn{3}{|c|}{ ادراك كلى درد } \\
\hline \multicolumn{3}{|r|}{ تحريك نقطه هوكو با سرما } \\
\hline$<\cdot / . .1$ & $-11 / \cdot 1$ & تحريك نقطه هوكو با كرما \\
\hline \multirow[t]{2}{*}{$<\cdot / \cdot .1$} & $-r \varphi / 11$ & 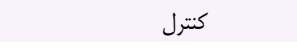 \\
\hline & & تحريك نقطه هوكو با ترما \\
\hline$<. / . .1$ & $-r \mu / . r$ & 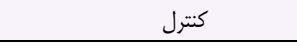 \\
\hline
\end{tabular}

به دنبال معنادار شدن اختلاف بين سه گروه، از آزمون تعقيبى براى

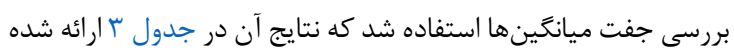

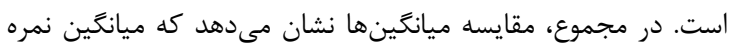

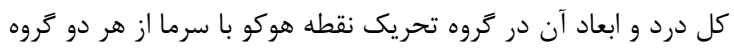

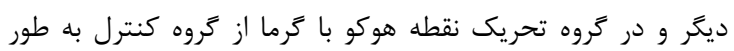

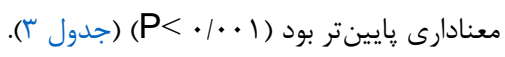

اين مطالعه با هدف مقايسه تأثير تحريك نقطه هوكو با سرما و كرما بر

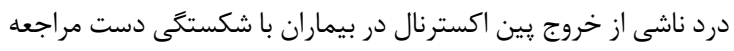

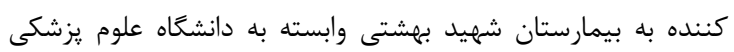

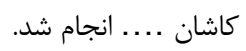

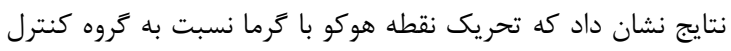

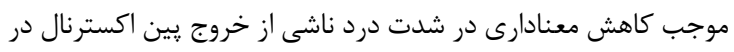

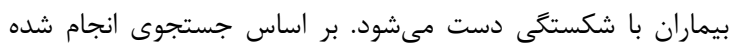

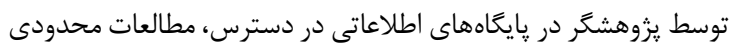

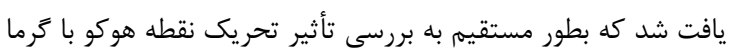

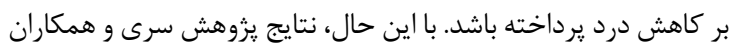

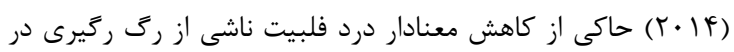




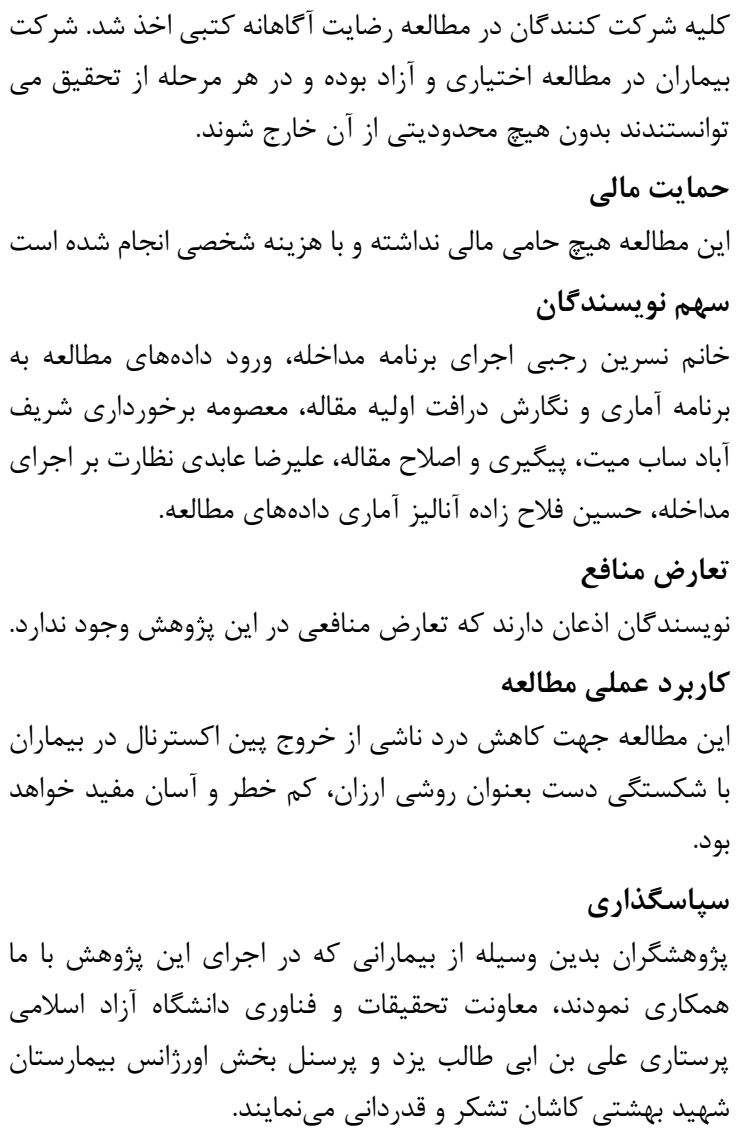

\section{References}

1. Chung KC, Spilson SV. The frequency and epidemiology of hand and forearm fractures in the United States. J Hand Surg Am. 2001;26(5):908-15. doi: 10.1053/jhsu.2001.26322 pmid: 11561245

2. Ameri M, Aghakhani K, Ameri E, Mehrpisheh S, Memarian A. Epidemiology of the upper extremity trauma in a traumatic center in Iran. Global J Health Sci. 2017;9(4):97-105. doi: 10.5539/gjhs.v9n4p97

3. Nakashian MN, Pointer L, Owens BD, Wolf JM. Incidence of metacarpal fractures in the US population. Hand (N Y). 2012;7(4):426-30. doi: 10.1007/s11552012-9442-0 pmid: 24294164

4. Commission UCPS. Division of hazard and injury data systems. National Electronic Injury Surveillance System: A Tool for Researchers; 2000.

5. Ryder S, Gorczyca JT. Routine removal of external fixators without anesthesia. J Orthop Trauma. 2007;21(8):571-3. doi: $\quad 10.1097 /$ BOT.0b013e31814b81bc pmid: 17805024

6. Rongieres M. Is the external fixator yet useful for treating fractures of the distal radius? Eur J Orthop Surg Traumatol. 2018;28(8):1495-7. doi: 10.1007/s00590018-2237-4 pmid: 29869273

7. Pommer A, Muhr G, David A. Hydroxyapatite-coated Schanz pins in external fixators used for distraction

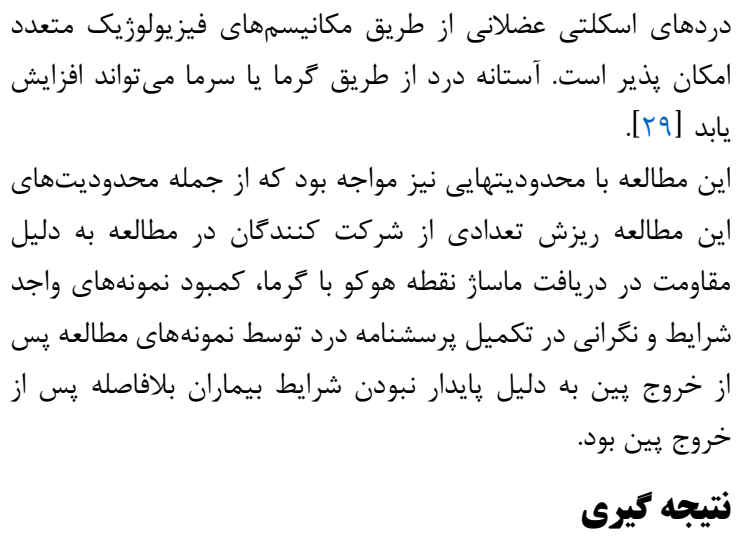

osteogenesis: a randomized, controlled trial. J Bone Joint Surg Am. 2002;84(7):1162-6. doi: 10.2106/00004623200207000-00011 pmid: 12107316

8. Bahrani B, DeKoven J. Not all Polysporin products contain lidocaine. CMAJ. 2018;190(3):E85. doi: 10.1503/cmaj. 733515 pmid: 29358205

9. Hindriati T. Effect of massage and relaxation of breathing against the duration of the phase I stage of labor active in the maternity hospital Jambi city 2015. J Bahana Kesehatan Masyarakat (Bahana J Pub Health). 2017;1(2):121-31.

10. Ibrahim HAF, Elgzar WTI, Hassan HE. Effect of warm compresses versus lubricated massage during the second stage of labor on perineal outcomes among primiparous women.

11. Yildirim E, Alan S, Gokyildiz S. The effect of ice pressure applied on large intestinal 4 on the labor pain and labor process. Complement Ther Clin Pract. 2018;32:25-31. doi: 10.1016/j.ctcp.2018.02.015 pmid: 30057052

12. Khosravan S, Atayee P, Mazloum Shahri SB, Mojtabavi SJ. Effect of Hugo's point massage with and without ice on vaccination-related pain in infants. J Hayat. 2018;24(1):719. 
13. Waters BL, Raisler J. Ice massage for the reduction of labor pain. J Midwife Women Health. 2003;48(5):317-21. doi: 10.1016/S1526-9523(03)00277-0

14. P BS, Khakha DC, Mahajan S, Gupta S, Agarwal M, Yadav SL. Effect of cryotherapy on arteriovenous fistula puncture-related pain in hemodialysis patients. Indian J Nephrol. 2008;18(4):155-8. doi: 10.4103/09714065.45290 pmid: 20142927

15. Pouraboli BAF, Rostami M, Jahani Y. Comparison the effect of two methods of acupressure and massage with ice on Huko point on pain intensity during IV insertion in pediatrics with thalassemia. Iranian J Pediatric Nurs. 2015;2(2):18-28.

16. Melzack R. The McGill pain questionnaire: major properties and scoring methods. Pain. 1975;1(3):277-99. doi: 10.1016/0304-3959(75)90044-5

17. Khosravi M, Moradi SH, Zendehdel K. Persian-McGill pain questionnaire translation, adaptation and reliability in cancer patients: a brief report. Tehran Univ Med J. 2013;71(1):53-8.

18. Sri Wahyuni N, Johan T, Yuswanto A. The efeectiveness of hot compress toward pain reduction due to phlebitis caused by intravenus line set up in Malang and Ponorogo, East-Java Indonesia. IOSR-J. 2014;3(4):28-31. doi: 10.9790/1959-03432831

19. Cluett ER, Burns E, Cuthbert A. Immersion in water during labour and birth. Cochrane Database Syst Rev. 2018;5:CD000111. doi: 10.1002/14651858.CD000111.pub4 pmid: 29768662

20. Mayer JM, Ralph L, Look M, Erasala GN, Verna JL, Matheson LN, et al. Treating acute low back pain with continuous low-level heat wrap therapy and/or exercise: a randomized controlled trial. Spine J. 2005;5(4):395-403. doi: 10.1016/j.spinee.2005.03.009 pmid: 15996609

21. Mayer JM, Mooney V, Matheson LN, Erasala GN, Verna JL, Udermann BE, et al. Continuous low-level heat wrap therapy for the prevention and early phase treatment of delayed-onset muscle soreness of the low back: a randomized controlled trial. Arch Phys Med Rehabil. 2006;87(10):1310-7. doi: 10.1016/j.apmr.2006.07.259 pmid: 17023239

22. Michlovitz S, Hun L, Erasala GN, Hengehold DA, Weingand KW. Continuous low-level heat wrap therapy is effective for treating wrist pain. Arch Phys Med Rehabil.
2004;85(9):1409-16. doi: 10.1016/j.apmr.2003.10.016 pmid: 15375809

23. Lee SL, Liu CY, Lu YY, Gau ML. Efficacy of warm showers on labor pain and birth experiences during the first labor stage. J Obstet Gynecol Neonatal Nurs. 2013;42(1):1928. doi: 10.1111/j.1552-6909.2012.01424.x pmid: 23167574

24. Nurcan Ç, Karadag M. Superficial heat and cold applications in the treatment of knee osteoarthritis. Osteoarthritis Progress Basic Res Treatment. 2015;26(23):249-64. doi: 10.5772/60534

25. Kaviani M, Ashoori M, Azima S, Rajaei Fard AR, Hadian Fard MJ. Comparing the effect of two methods of acupressure and ice massage on the pain, anxiety levels and labor length in the point LI-4. SSU J 2012;20(2):22028.

26. Fadeel NA. Effect of ice cold massage and acupressure on labor pain and labor duration. Randomi Control Trial J Natural Sci Res. 2015;5(22):251-60.

27. Faroukh A, Pouraboli B, Rostami M, Jahani Y. The effect of Hoku point massage with ice on venipuncture pain in children with Thalassemia. Manager J Nurs. 2015;5(4):13-9.

28. Abbasi Teshnizi M, Kianinejad A, Gandomkar F. Effect of applying Ice bag on pain intensity associated with chest tube removal after cardiac surgery. Horizon Med Sci. 2012;18(3):109-14.

29. Garra G, Singer AJ, Leno R, Taira BR, Gupta N, Mathaikutty B, et al. Heat or cold packs for neck and back strain: a randomized controlled trial of efficacy. Acad Emerg Med. 2010;17(5):484-9. doi: 10.1111/j.15532712.2010.00735.x pmid: 20536800

30. Curković B, Vitulić V, Babić-Naglić D, Dürrigl T. The influence of heat and cold on the pain threshold in rheumatoid arthritis. Zeitschrift fur Rheumatologie. 1993;52(5):289-91.

31. Can HO, Saruhan A. Evaluation of the effects of ice massage applied to large intestine 4 (hegu) on postpartum pain during the active phase of labor. Iran J Nurs Midwife Res. 2015;20(1):129-38.

32. Safdari Dehcheshmeh F, Delaram M, Salehian T, Moradi MT, Rahimi Madiseh M, Aliakbari F. Relief of labor pain by ice massage of the hand. Zahedan J Res Med Sci. 2009;11(1):23-30. 